\title{
Three-dimensional echocardiographic assessment of rhabdomyoma in a newborn: the merit of three dimension over two dimension
}

Keywords: Echocardiography; cardiac tumour; three dimensional

Received: 6 June 2011; Accepted: 19 August 2011; First published online: 15 September 2011

\section{Dear Editor,}

Ladombarda et al's article ${ }^{1}$ attracted our attention. They showed for the first time three-dimensional echocardiographic images of rhabdomyoma complicated with tuberous sclerosis in an infant. In their case, observation by two-dimensional echocardiography, if made cautiously, would have revealed almost all features of the tumours and the cardiac functional morphology. Furthermore, does three dimension have advantages over two dimension in assessing infantile cardiac tumours; if it does what are the advantages? We believe that three-dimensional echocardiography may have three possible advantages over two-dimensional echocardiography as far as infantile cardiac assessment is concerned.

First, three-dimensional echocardiography may definitely identify the number of tumours in a small infantile heart occupied by multiple tumours. In our case, ${ }^{2}$ although two-dimensional echocardiography clarified the presence of multiple cardiac tumours in a foetus from mother with tuberous sclerosis, the detailed features were not identified. Soon after birth, two-dimensional echocardiography confirmed six intra-cardiac tumours. The data set for three-dimensional echocardiography was acquired. Further analysis of the data set revealed another small left ventricular tumour just adjacent to the aortic valve, the seventh tumour, the presence of which was not determined by two-dimensional echocardiography. Rapid movement of the aortic valve may have prevented us from finding this seventh tumour by two-dimensional echocardiography. A perspective image of this seventh tumour enabled us to confirm the absence of aortic outflow

Correspondence to: Prof. S. Matsubara, MD, PhD, Department of Obstetrics and Gynecology and Perinatal Education Center, Jichi Medical University, 3311-1 Shimotsuke, 329-0498 Tochigi, Japan. Tel: +81 28558 7375; Fax: +81285448505; E-mail: matsushi@jichi.ac.jp obstruction, and also to decide the expectant management.

Second, just one three-dimensional capture may give a lot of information, reducing the time required, which will benefit an infant who cannot lie still for a long time. This may reduce the chance for sedation.

Third, three-dimensional echocardiography will enable a "bird's eye view", and thus a "surgeon's eye view". This will be a great advantage for surgeons, especially paediatric cardiac surgeons.

We must admit the possible demerits of the threedimensional echocardiography. Low resolution is one of the major disadvantages of three dimension, which should be overcome in future. Aside from the general discussion regarding the merits/demerits of threedimensional echocardiography versus two-dimensional echocardiography in cardiac assessment, however, we believe that an infant with cardiac tumour will be greatly benefited by three-dimensional echocardiography. The combined use of two-dimensional and three-dimensional echocardiography may be practical. However, three-dimensional echocardiography may play a greater role in infants in assessing cardiac tumour.

\section{Daisuke Matsubara Department of Pediatrics \\ Shigeki Matsubara Jichi Medical University Shimotsuke, Tochigi, Japan} Department of Obstetrics and Gynecology and Perinatal Education Center

\section{References}

1. Labombarda F, Maragnes P, Saloux E. Three-dimensional echocardiographic assessment of rhabdomyoma in a newborn. Cardiol Young 2010; 20: 565-566.

2. Matsubara D, Matsubara S, Kikuchi Y, et al. Real-time threedimensional echocardiographic assessment of neonatal cardiac tumors. Jichi Med Univ J 2011; 34: (in press). 